\title{
Pacific
}

Journal of

Mathematics

\section{QUASI-EXCEPTIONAL DOMAINS}

ALEXANDRE EREMENKO AND ERIK LUNDBERG 


\title{
QUASI-EXCEPTIONAL DOMAINS
}

\author{
AlEXANDRE EREMENKO AND ERIK LUNDBERG
}

\begin{abstract}
Exceptional domains are domains on which there exists a positive harmonic function, zero on the boundary and such that the normal derivative on the boundary is constant. Recent results classify (under some mild additional assumptions) exceptional domains as belonging to either a certain one-parameter family of simply periodic domains or one of its scaling limits.

We introduce quasi-exceptional domains by allowing the boundary values to be different constants on each boundary component. This relaxed definition retains the interesting property of being an arclength quadrature domain, and also preserves the connection to the hollow vortex problem in fluid dynamics. We give a partial classification of such domains in terms of certain abelian differentials. We also provide a new two-parameter family of periodic quasi-exceptional domains. These examples generalize the hollow vortex array found by Baker, Saffman, and Sheffield. A degeneration of regions of this family provides doubly connected examples.
\end{abstract}

\section{Introduction}

A domain $D \in \mathbb{R}^{n}$ is called exceptional if there is a positive function $u$ (called a roof function) harmonic in $D$, zero on the boundary, and with

$$
\frac{\partial}{\partial n} u(z)=1, \quad z \in \partial D,
$$

where the differentiation is along the normal pointing inwards into $D$ and it is assumed that the boundary is smooth. Evident examples are exteriors of balls and half-spaces. For $n>2$, the only other known examples are cylinders whose base is an exceptional domain in $\mathbb{R}^{2}$. If the smoothness assumption on the boundary is dropped, then there are also certain cones in higher dimensions and pathological "non-Smirnov" examples in the plane [Khavinson et al. 2013].

The problem of describing of all exceptional domains in the plane was stated in [Hauswirth et al. 2011] and settled in [Khavinson et al. 2013] under a topological

Supported by NSF grant DMS-1361836.

MSC2010: primary 30C20, 35R35; secondary 76B47, 31A05.

Keywords: quadrature domains, hollow vortices, elliptic functions, abelian differentials. 
assumption which was removed in [Traizet 2014a] using an unexpected correspondence with minimal surfaces. The first nontrivial example was given in [Hauswirth et al. 2011]. This example appeared in another context, related to fluid dynamics, in [Longuet-Higgins 1988]. A second nontrivial example was noticed in [Khavinson et al. 2013] and [Traizet 2014a]. This example had also appeared previously in studies of fluid dynamics [Baker et al. 1976] (see also [Crowdy and Green 2011]).

Let us introduce quasi-exceptional domains, by relaxing the definition to allow the Dirichlet condition to be a different constant on each boundary component. Thus, a domain $D \in \mathbb{R}^{n}$ is called quasi-exceptional if there is a positive harmonic function $u$ in $D$ which is constant on each boundary component (but not necessarily the same constant) and the Neumann condition (1) holds. We will continue to call $u$ a roof function. Again, we assume that each component of the boundary is smooth.

Added in press: In an interesting preprint, Martin Traizet [2014b] has considered an even more general problem, allowing the Neumann data to take different signs on different components. As with the current paper, that work is motivated by the hollow vortex problem from fluid dynamics. Extending [Traizet 2014a], a correspondence to minimal surfaces is given in that work, and techniques from minimal surface theory are used to produce new examples.

We summarize several interesting aspects of exceptional domains. These statements all hold true for quasi-exceptional domains.

- Fluid dynamics: As noted above, the two nontrivial examples first appeared in fluid dynamics [Longuet-Higgins 1988; Baker et al. 1976]. In general, one can interpret exceptional domains in terms of a hollow vortex problem. The level lines of $u$ can be interpreted as stream lines of a two-dimensional stationary flow of ideal fluid, and condition (1) expresses the fact that the pressure is constant on the boundary. Such conditions may exist if the components of the complement of $D$ are air bubbles in the surrounding liquid. Notice that the rotation of the fluid around all bubbles corresponding to exceptional domains is in the same direction because $\partial u / \partial n>0$ on the boundary.

- Quadrature domains [Gustafsson 1987]: Exceptional domains provide examples of arclength null-quadrature domains, that is, domains for which integrals with respect to arclength over $\partial D$ of every analytic function in the Smirnov class $E^{1}(D)$ vanish.

- Differentials on Riemann surfaces: By way of the connection to quadrature domains, the study [Gustafsson 1987] indicates a connection to half-order differentials. We make use of abelian differentials in Section 4 below. 
- The Schwarz function of a curve: In [Khavinson et al. 2013], it was noticed that the function $u(z)$ satisfies

$$
\partial_{z} u(z)=\sqrt{-S^{\prime}(z)},
$$

where $S(z)$ is the Schwarz function of $\partial \Omega$ and $\partial_{z}=\frac{1}{2}\left(\partial_{x}-i \partial_{y}\right)$ is the CauchyRiemann operator.

- Minimal surfaces: Traizet [2014a] established a nontrivial correspondence between exceptional domains and a special type of minimal surfaces called "minimal bigraphs". In [Traizet 2014b], this correspondence was extended to quasi-exceptional domains, but the minimal surfaces in that case need not be embedded. This prevents applying the results on complete embedded minimal surfaces that were used in [Traizet 2014a] to classify exceptional domains.

The classification results for exceptional domains of finite connectivity show that they are quite restricted; all examples can be conformally mapped onto a disk by elementary functions.

Problem A. Classify quasi-exceptional domains.

We begin to address this problem below, give a partial classification of periodic and finitely connected exceptional domains, and provide new periodic and doubly connected examples described in terms of elliptic functions. First, we explain the relation to arclength null-quadrature domains.

\section{Arclength null-quadrature domains}

A bounded domain $D \subset \mathbb{C}$ is a quadrature domain if it admits a formula expressing the area integral of every function $f$ analytic and integrable in $D$ as a finite sum of weighted point evaluations of the function and its derivatives, i.e.,

$$
\int_{D} g(z) d A(z)=\sum_{m=1}^{N} \sum_{k=0}^{n_{m}} a_{m, k} g^{(k)}\left(z_{m}\right),
$$

where the $z_{m}$ are distinct points in $D$ and the $a_{m, k}$ are constants independent of $g$.

A (necessarily unbounded) domain $D \subset \mathbb{C}$ is called a null-quadrature domain (NQD) if the area integral of every function $g$ analytic and integrable in $D$ vanishes:

$$
\int_{D} g(z) d A(z)=0 .
$$

M. Sakai [1981] completely classified NQDs in the plane.

Following [Khavinson et al. 2013], we refer to a domain $D \subset \mathbb{C}$ as an arclength null-quadrature domain (ALNQD) if the integral over $\partial D$ of every function $g$ in 
the Smirnov class $E^{1}(D)$ vanishes (in the case $\infty$ is an isolated point on $\partial D$, we take the restricted class of functions $g(z) \in E^{1}(D)$ vanishing at infinity):

$$
\int_{\partial D} g(z) d s(z)=0 .
$$

The Smirnov class $E^{1}(D)$ is not the same as the Hardy space $H^{1}(D)$. Namely, a function $g$ analytic in $D$ is said to belong to $E^{1}(D)$ if there exists a sequence of domains $D_{1} \subset D_{2} \subset \cdots$, with $\bigcup_{k} D_{k}=D$, and with rectifiable boundaries, such that:

$$
\sup _{k} \int_{\partial D_{k}}|g(z)||d z|<\infty \text {. }
$$

One may also define quadrature domains in higher dimensions using a test class of harmonic functions, but we will restrict ourselves to the case of $n=2$ dimensions.

Inspired by the successful classification of NQDs [Sakai 1981], the problem of classifying ALNQDs was suggested in [Khavinson et al. 2013]. We pose this problem again while stressing that it does not reduce to the classification of exceptional domains (whereas it might reduce to classification of quasi-exceptional domains).

Problem B. Classify ALNQDs.

The following proposition shows that quasi-exceptional domains are ALNQDs. Thus, the new examples (described in the last section) of quasi-exceptional domains also provide new ALNQDs. Problem B is closely related to Problem A, and if the converse of the proposition is true then the two problems are equivalent.

Proposition 1. If $D$ is a quasi-exceptional domain, then $D$ is an ALNQD.

Proof. Consider the complex analytic function $F(z)=u_{x}-i u_{y}$, where $u$ is the roof function. We will need the following claim, which is proved in the next section (see Lemma 2).

Claim. The roof function $u$ of $D$ satisfies $\nabla u(z)=O(1)$ in $D$, so $F(z)$ is bounded.

Suppose that $g$ is in the Smirnov space $E^{1}(D)$. Using the fact that $d s=i F(z) d z$,

$$
\int_{\partial D} g(z) d s=\int_{\partial D} i g(z) F(z) d z .
$$

As $F$ is bounded, $g F \in E^{1}(D)$. If $\infty$ is not an isolated boundary point, then the integral equals zero by Cauchy's theorem.

If $\infty$ is an isolated boundary point, then we have $u(z)=\log |z|+$ const $+O(1 / z)$, so $F(z)=O(1 / z), z \rightarrow \infty$. Now $g(\infty)=0$, so $F g$ has a zero of order at least 2 at $\infty$, and the integral is zero again. 


\section{A potential theoretic restriction on the roof function}

We restrict ourselves to the case $n=2$, and assume that the order of connectivity of $D$ is finite, or that the roof function $u$ is periodic and a fundamental region for $D$ has finite connectivity.

Recall that a Martin function is a positive harmonic function $M$ in a domain $\Omega$ with the property that for any positive harmonic function $v$ in $\Omega$ the condition $v \leq M$ implies that $v=c M$, where $c>0$ is a constant. (Often, Martin functions are called minimal harmonic functions - see [Heins 1950].) Martin functions on finitely connected domains are simply Poisson kernels evaluated at points of the Martin boundary, the boundary under Carathéodory compactification (prime ends) of the domain (see [Brelot 1971]).

Any domain $D$ of finite connectivity in $\mathbb{C}$ is conformally equivalent to a circular domain $\Omega$. A circular domain is a domain whose boundary components are points or circles. For a circular domain, a Martin function $M$ can be of two types:

(a) There is a component of $\partial \Omega$ which is a single point $z_{0}$, and $M$ is proportional to the Green function of $\Omega \cup\left\{z_{0}\right\}$ with the singularity at $z_{0}$.

(b) There is a point $z_{0} \in \partial \Omega$ which is not a component of $\partial \Omega$, and $M$ has boundary value zero at all points of $\partial \Omega \backslash\left\{z_{0}\right\}$. The local behavior in this case is like $-\operatorname{Im}(1 / z)$ in the upper half-plane near 0 .

Let $D$ be an exceptional domain, and $u$ a harmonic function with the property (1). The following result was proved for exceptional domains by the current first author, but was communicated in [Khavinson et al. 2013, Theorem 4.2]. Here we repeat the proof with minor adjustments.

Lemma 2. The roof function u of a quasi-exceptional domain satisfies the equation $\nabla u(z)=O(1)$ in D. Moreover, $u$ is the sum of a bounded harmonic function and at most two Martin functions.

Proof. We follow the second part of the proof from [Khavinson et al. 2013]. Let $R>0$, and consider an auxiliary function

$$
w_{R}=\frac{|\nabla u|}{u+R} .
$$

A direct computation shows that

$$
\Delta \log w_{R}=w_{R}^{2},
$$

and $w_{R}(z)=1 /\left(c_{k}+R\right) \leq 1 / R$ for $z \in \partial D$, where $c_{k} \geq 0$ are the constants taken in the Dirichlet condition. We claim that

$$
w_{R}(z) \leq 2 / R, \quad z \in D,
$$


from which the result follows by letting $R \rightarrow \infty$, which gives $|\nabla u| \leq 2$ in $D$.

Suppose, contrary to (7), that $w_{R}\left(z_{0}\right)>2 / R$ for some $z_{0} \in D$. Let

$$
v(z)=\frac{2 R}{R^{2}-\left|z-z_{0}\right|^{2}}, \quad z \in B\left(z_{0}, R\right)=\left\{z:\left|z-z_{0}\right|<R\right\} .
$$

Obviously, $v(z) \geq 2 / R$. A computation reveals that $\Delta \log v=v^{2}$. Let

$$
K=\left\{z \in D \cap B\left(z_{0}, R\right): w_{R}(z)>v(z)\right\} .
$$

We have $z_{0} \in K$, since $v\left(z_{0}\right)=2 / R$. Let $K_{0}$ be the component of $K$ containing $z_{0}$. Then we have $w_{R}(z)=v(z)$ on $\partial K_{0}$, since $w_{R}(z)<v(z)$ on $\partial D \cap B\left(z_{0}, R\right)$ while $v(z)=+\infty$ on $\partial B\left(z_{0}, R\right)$. On the other hand,

$$
\Delta\left(\log w_{R}-\log v\right)=w_{R}^{2}-v^{2}>0 \quad \text { in } K_{0} .
$$

So the subharmonic function $\log w_{R}-\log v$ is positive in $K_{0}$ and vanishes on the boundary - a contradiction.

This proves that $\nabla u=O(1)$. In order to see the second statement, we note that $\nabla u=O(1)$ implies that $u(z)=O(|z|)$ has order 1 . The result then follows by first solving the Dirichlet problem (with a bounded function) having the same boundary values as $u$; subtracting this function, one may then apply [Kjellberg 1950, Theorem II].

\section{Partial classification in terms of abelian differentials}

Let $D$ be a $\mathrm{QE}$ domain of one of the following types:

Type I: $D$ is finitely connected.

Type II: $D / \Gamma$ is finitely connected, where $\Gamma$ is the group of transformations $z \mapsto z+n \omega$, and $u(z+\omega)=u(z)$ for some $\omega \in \mathbb{C} \backslash\{0\}$. We call this the periodic case. (As above, $u$ is the roof function.)

In this section we give a classification of QE domains of these two types in terms of abelian differentials of a compact Riemann surface with an anticonformal involution.

If $D$ is of type $I$, and $\infty$ is an isolated boundary point, then $D^{\prime}=D \cup\{\infty\}$ is conformally equivalent to some bounded circular domain $\Omega$, and we suppose that $p \in \Omega$ corresponds to $\infty$. If $\infty$ is not isolated, we put $D^{\prime}=D$, and $\Omega$ is a bounded circular domain conformally equivalent to $D^{\prime}$. In any case, we have a conformal map $\phi: \Omega \rightarrow D^{\prime}$, which may have at most one simple pole at $p \in \Omega$.

If $D$ is of type II, let $G=D / \Gamma$. The Riemann surface $G$ is a finitely connected domain on the cylinder $\mathbb{C} / \Gamma$; this cylinder is conformally equivalent to the punctured plane, and we identify it with $\mathbb{C}^{*}$. Then $G \subset \mathbb{C}^{*}$ must have one or two punctures of $\mathbb{C} / \Gamma$ as isolated boundary points, and we denote by $G^{\prime}$ the union of $G$ with 
these isolated boundary points. Then $G^{\prime}$ is conformally equivalent to a bounded circular domain of finite connectivity $\Omega$ in which there are one or two points $a$ and $b$ corresponding to the added punctures. We have a multivalued conformal map $\phi: \Omega \rightarrow D$.

The points $a$ and $b$ are logarithmic singularities of $\phi$.

We pull back $u$ on $\Omega$; i.e., set $v=u \circ \phi$. As $u$ is periodic, $v$ is a single-valued positive harmonic function on $\Omega \backslash\{a, b\}$. Consider the differential on $\Omega$

$$
d v=v_{z} d z=\frac{1}{2}\left(v_{x}-i v_{y}\right)(d x+i d y)=g(z) d z .
$$

This is well-defined on $\Omega: g$ is a single-valued meromorphic function in $\Omega$ with at most simple poles at $p$ or $a$ and $b$. Indeed, for a positive harmonic function, an isolated singularity is either removable or logarithmic. In the second case the gradient has a simple pole.

Next, we extend $v$ as a multivalued function to a compact Riemann surface $S$. Let $\Omega^{\prime}$ be the mirror image of $\Omega$; we glue it to $\Omega$ in the standard way (along each circular boundary component) and obtain a compact Riemann surface $S$. We denote by $\sigma: z \mapsto z^{*}$ the anticonformal involution which fixes the boundary components of $\Omega$. The Riemann surface $S$ is of genus $g$, and the involution $\sigma$ has fixed set corresponding to $\partial \Omega$, which consists of $n=g+1$ ovals. Such involutions are called involutions of maximal type, meaning that the complement of the fixed set of the involution consists of two regions homeomorphic to planar regions.

Each branch of $v$ is constant on each boundary component, so it extends through this boundary component by reflection to the double $S$ of $\Omega$. The extensions of various branches of $v$ through different boundary components do not match: they differ by additive constants. On the other hand, the differential $d v$ is well-defined on the double. Namely,

$$
(d v)^{*}=-d v,
$$

where $*$ is the action of involution on differentials. Thus we have a meromorphic differential $d v$ on $S$.

Choose a basis of 1-homology in $S$ so that the $A$-loops are simple closed curves in $\Omega$, each homotopic to one boundary component of $\Omega$, and the $B$ loops are dual to the $A$-loops. For type I, all periods over $A$-loops are purely imaginary, because

$$
v=\operatorname{Re} \int d v
$$

is single-valued. For type II, these periods are imaginary except those which correspond to simple loops around one pole, $a$ or $b$. 
Now we discuss $\phi$, or better, the differential $d \phi=\phi^{\prime}(z) d z$. We have, from the condition that our domain is quasi-exceptional,

$$
2|d v|=|d \phi| \quad \text { on } \partial \Omega .
$$

The ratio of two differentials is a function. So we have a meromorphic function $B$ on $\Omega$ such that

$$
2 B d v=d \phi .
$$

This function has absolute value 1 on $\partial \Omega$. Therefore, it extends to $S$ by symmetry. It has no zeros in $\Omega$ because $d \phi$ has no zeros. Its poles in $\Omega$ must match the zeros of $d v$, because $d \phi$ is zero-free (indeed, $\phi$ is univalent). In fact, $B$ is a meromorphic function on $S$. To justify this claim when $d v$ has a singularity on $\partial \Omega$, we observe that this singularity is removable for $B$, which follows from the next lemma:

Lemma 3. Consider the equation

$$
\phi^{\prime}=B h,
$$

where $h$ is meromorphic in a neighborhood $V$ of $0, B$ is holomorphic and zero-free in $V \backslash\{0\},|B(z)|=1$ for $z \in V \cap \mathbb{R} \backslash\{0\}$, and $\phi$ is univalent in $\{z \in V: \operatorname{Im} z>0\}$. Then the singularity of $B$ at 0 is removable.

Before proving the lemma, we note that in order to apply it in our setting we compose $B$ with a linear fractional transformation that sends $V$ to a neighborhood of the singularity we wish to remove such that the real line is mapped to the circular boundary component with 0 sent to the singularity.

Proof. In order to prove this by contradiction, assume that 0 is an essential singularity of $B$. By symmetry we have $B(\bar{z})=1 / \overline{B(z)}$. We claim that there exists a sequence $z_{k} \rightarrow 0$ such that

$$
\liminf _{k \rightarrow \infty}\left|z_{k} \log \right| B\left(z_{k}\right)||>0 \text {. }
$$

Indeed, suppose that this is not so. Then $\log |B(z)|=o\left(z^{-1}\right)$, and the PhragménLindelöf theorem (see, for example, [Levin 1980, Chapter I, Theorem 22]) implies that $B$ has a limit as $z \rightarrow 0$. By choosing a subsequence and using symmetry, we can find a sequence in the upper half-plane with the property

$$
\liminf _{k \rightarrow \infty}\left|z_{k}\right| \log \left|B\left(z_{k}\right)\right|>0
$$

or with the property

$$
\liminf _{k \rightarrow \infty}\left|z_{k}\right| \log \left|B\left(z_{k}\right)\right|<0
$$


Distortion theorems for univalent functions imply that

$$
c(\operatorname{Im} z)^{3} \leq\left|\phi^{\prime}(z)\right| \leq C(\operatorname{Im} z)^{-3} .
$$

In addition to this, we have, for some integer $m$,

$$
c|x|^{m} \leq\left|\phi^{\prime}(x)\right| \leq C|x|^{m}, \quad z \in V \cap \mathbb{R},
$$

because $h$ is meromorphic and $|B(x)|=1$ for $z \in V \cap \mathbb{R}$. Taking $n=\max \{3,-m\}$, we obtain that the subharmonic function $u(z)=\log ^{+}\left|z^{n} \phi^{\prime}(z) / C\right|$ satisfies $u(x)=0$ for $x \in V \cap \mathbb{R}$, and $u\left(r e^{i \theta}\right) \leq \psi(\theta)$, where $\psi(\theta)=-3 \log \sin \theta$ for $\theta \in(0, \pi)$. As

$$
\int_{0}^{\pi} \psi(\theta) d \theta<\infty
$$

we can apply Carleman's "log log" theorem [Carleman 1926; Rashkovskii 2009], and conclude that $u$ is bounded from above in the intersection of $V$ with the upper half-plane. This contradicts (11). If (12) holds, one applies the same argument to $1 / \phi^{\prime}$. This completes the proof of the lemma.

We can thus restate the problem of finding QE domains (under the restrictions we impose) as follows:

Proposition 4. All QE domains of types I and II are parametrized by triples $(S, d \omega, B)$, where $S$ is a compact Riemann surface with an involution of maximal type, $d \omega$ is a meromorphic differential that enjoys the symmetry property (8), and $B$ is a function that has the symmetry property

$$
B^{*}(z):=\overline{B\left(z^{*}\right)}=1 / B(z)
$$

and has poles at the zeros of $d \omega$ on one half of $S$, that is, in $\Omega$. There is an additional condition: that

$$
\phi=2 \int B d \omega
$$

is globally univalent and single-valued in type I, and single-valued except the residues in type II.

To recover $D$ from a triple $(S, d w, B)$, one takes one of the components $\Omega \subset S$ complementary to the fixed set of the involution. Then $D=\phi(\Omega)$, where $\phi$ is defined in (15).

In order to check the condition on the global univalence of $\phi$, it is sufficient to verify that periods of $d \omega / B$ are zero on the boundary curves, and that these boundary curves are mapped by $\phi$ injectively.

The following is a general conclusion:

Proposition 5. The boundary of a quasi-exceptional domain of type I or type II is parametrized by an abelian integral. 
Next we provide a partial classification of quasi-exceptional domains in terms of the data stated in the above formulation.

Theorem 6. The differential $d v$ has either two or four poles on $S$, counting multiplicity. Moreover, if $d v$ has two poles in $S$, then $D$ is the complement of either a disk or a half-plane.

Remark. If $B \neq$ const, then $1 / B$ is an Ahlfors function of $\Omega$.

Proof of Theorem 6. Let us first show that $d v$ has some poles. Otherwise, $d v$ is holomorphic, and thus $u$ is bounded. Let $z_{1} \in \partial D$ and $z_{2} \in \partial D$ be the points where $u$ assumes its maximal and minimal values. Then $d u / d n$ has opposite signs at these two points, which contradicts (1).

The differential $d v$ has at most simple poles at $p, a$, and $b$ (whichever of these points are present) and at their images $\sigma p, \sigma a$, and $\sigma b$. In addition it may have double poles on $\partial \Omega$. The total number of poles (without multiplicity) in $\bar{\Omega}$ is at most two by Lemma 2. Thus, on $S$ the differential $d v$ has two or four poles, counting multiplicity.

Notice that $v$ is constant on each boundary component, so the gradient is perpendicular to the boundary $\partial \Omega$, so the total rotation of this gradient as we traverse the boundary is the same as the total rotation of the tangent vector to the boundary. This is equal to $2 \pi(2-n)$ because the outer boundary component is traversed counterclockwise and the rest clockwise, as parts of the boundary of $\Omega$. So $v_{z}$, which is conjugate to the gradient, rotates $n-2$ times.

From this we can conclude how many zeros $d v$ has in $\Omega$. The number $N$ of zeros of $d v$ in $\Omega$ satisfies

$$
n-2=N-(\text { the number of poles in } \Omega),
$$

where a double pole on $\partial \Omega$ is counted as a single pole in $\Omega$. This formula is well known.

Suppose that $d v$ has exactly two poles, counting multiplicity. This can occur in one of three ways:

Case (1): $d v$ has a simple pole at $p$ in $\Omega$.

Case (2): $d v$ has one double pole at $z_{0} \in \partial \Omega$.

Case (3): $d v$ has a simple pole at $a$ in $\Omega$ (and $b$ does not exist).

If Case (1) holds, then $\infty$ is an isolated point on $\partial D$, and, by Proposition $1, D$ is an arclength quadrature domain with quadrature point at $\infty$. It now follows from [Gustafsson 1987, Remark 6.1] that $D$ is the exterior of a disk.

In Case (2), we will show that $B$ is constant. First note that $d \phi$ has a double pole at $z_{0}$, so $B$ does not have a zero or a pole at $z_{0}$. Since $\phi$ is a conformal map, it follows from (9) that $B$ has no zeros and $N$ poles in $\Omega$ (located at the zeros of $d v$ ). 
Assume for the sake of contradiction that $B$ is not constant. By Lemma 3, $B$ is meromorphic in $S$, and, by Lemma $2,1 /|B|$ is bounded by a constant in $\Omega$. Since $|B|=1$ on $\partial \Omega, B$ thus maps $\Omega$ to the exterior of the unit disk and maps each of the $n$ components of $\partial \Omega$ to the unit circle. This implies that $B$ has at least $n$ poles in $\Omega$. Combined with (16), this gives the contradiction $N=n-1 \geq n$. We conclude that $B$ is constant, which implies that the gradient of the roof function is constant. Thus, the roof function is linear, and $D$ is a half-plane.

In Case (3), the behavior of $\phi$ at point $a$ is logarithmic, so $d \phi$ has a simple pole at $a$ and $B$ does not have a zero or a pole at $a$. Arguing as before, we conclude that $B$ is constant and that $D$ is a half-plane.

Corollary 7. The only $Q E$ domains with compact boundary are exteriors of disks, and the only $Q E$ domains of types I or II with one unbounded boundary component are half-planes.

If $D$ is a quasi-exceptional domain that is not a disk or half-plane, then $d v$ has four poles and, more precisely, we have the following two possibilities:

$D$ is of type I: $d v$ has two double poles on $\partial \Omega$. This implies that the boundary $\partial D$ consists of two simple curves tending to $\infty$ in both directions and $n-1$ bounded components. The unbounded components are the $\phi$-images of two arcs of one boundary circle of $\Omega$ which contains both singularities of $\phi$ and $v$.

$D$ is of type II: $d v$ has two simple poles in $\Omega$. In this case $D$ must be periodic, all components of $\partial D$ are compact, and there are $n$ such components per period.

Note that the possibility that $d v$ has one simple pole in $\Omega$ and one double pole on $\partial \Omega$ is excluded by Lemma 2 : it is easy to see that in this case the number of Martin functions in the decomposition of $u$ would be infinite.

We have thus described possible topologies of the QE domains satisfying the assumptions stated in the beginning of this section.

In the next section we construct the examples of types I and II with $S$ of genus 1 . We conjecture that there exist QE domains of types I and II with $S$ of any genus.

\section{New examples}

Description of our examples requires elliptic functions (all known exceptional domains can be parametrized by elementary functions).

Example of type I. Let $G$ be the rectangle with vertices $\left(0,2 \omega_{1}, 2 \omega_{1}+\omega_{3}, \omega_{3}\right)$, where $\omega_{1}=2 \omega, \omega>0$, and $\omega_{3}=\omega^{\prime}$, where $\omega^{\prime} \in i \mathbb{R}, \omega^{\prime} / i>\omega$. Let $G^{\prime}$ be the reflection of $G$ in the real line. The union of $G, G^{\prime}$ and the interval $\left(0,2 \omega_{1}\right)$ make a fundamental domain of the lattice $\Lambda$ generated by $2 \omega_{1}, 2 \omega_{3}$. 
Let us consider the $\omega_{1}$-periodic positive harmonic function $h$ in $G$ which is zero on the horizontal segments of the boundary $\partial G$, except for one singularity per period, at 0 , where it behaves in the following way:

$$
h(z) \sim-\operatorname{Im}(1 / z), \quad z \rightarrow 0 .
$$

Note that the existence of $h$ is clear as it can be expressed (through conformal mapping) in terms of the Poisson kernel of a ring domain.

The function $h$ has two critical points in $G$ : at $w_{1}$ and $w_{2}$ with $\operatorname{Re} w_{1}=\omega_{1} / 2$ and $\operatorname{Re} w_{2}=3 \omega_{1} / 2$, while the imaginary parts of $w_{1}$ and $w_{2}$ are equal. Let us choose real constants $c_{1}$ and $c_{2}$ such that $v=2\left(h+c_{1} y\right)+c_{2}$ is a positive harmonic function with critical points $\omega_{1} / 2+\omega_{3} / 2$ and $3 \omega_{1} / 2+\omega_{3} / 2$. The existence of such constants $c_{1}$ and $c_{2}$ is evident by continuity.

The $z$-derivative $\partial_{z} v=\left(v_{x}-i v_{y}\right) / 2$ is an elliptic function with periods $\omega_{1}, 2 \omega_{3}$, and thus also elliptic with periods $\Lambda$. Asymptotics near 0 show that $\partial_{z} v \sim-i / z^{2}$, and, as this function has only one pole per period (with respect to the parallelogram $\left.\omega_{1}, 2 \omega_{3}\right)$, we have $\partial_{z} v=-i \wp+i c_{0}$, where $\wp$ is the Weierstrass function corresponding to the lattice $\left(\omega_{1}, 2 \omega_{3}\right)$. Zeros of $\partial_{z} v$ in $G \cup G^{\prime}$ are $\omega_{1} / 2+\omega_{3} / 2,3 \omega_{1} / 2+\omega_{3} / 2$ and their complex conjugates in $G^{\prime}$.

Let $B$ be an elliptic function with periods $2 \omega_{1}, 2 \omega_{3}$ having simple poles at $\omega_{1} / 2+\omega_{3} / 2,3 \omega_{1} / 2+\omega_{3} / 2$, and zeros at complex conjugate points. Such a function exists by Abel's theorem: the sum of zeros minus the sum of poles equals $-2 \omega_{3}$. This function is unique up to a constant factor. By symmetry, $B(\bar{z})=c / \overline{B(z)}$, so on the real line $|B(x)|^{2}=c$ and we can choose the constant factor in the definition of $B$ so that $c=1$. Thus

$$
|B(x)|=1, \quad x \in \mathbb{R} .
$$

Then we have $B\left(x+\omega_{3}\right) \overline{B\left(x-\omega_{3}\right)}=1$, but by periodicity we also have $B\left(x+\omega_{3}\right)=$ $B\left(x-\omega_{3}\right)$, thus $\left|B\left(x+\omega_{3}\right)\right|=1$. So

$$
|B(z)|=1 \quad \text { on the horizontal segments of } \partial G .
$$

Now we consider the function

$$
F=\frac{\partial v}{\partial z} B=\left(-i \wp+i c_{0}\right) B
$$

This function $F$ is holomorphic and zero-free in $G$ (the zeros of $\partial v / \partial z$ in $G$ are exactly canceled by the poles of $B$ ). Let us show that

$$
\int_{0}^{2 \omega_{1}} F(x+i y) d x=0, \quad y \in\left(0, \omega_{3}\right) .
$$




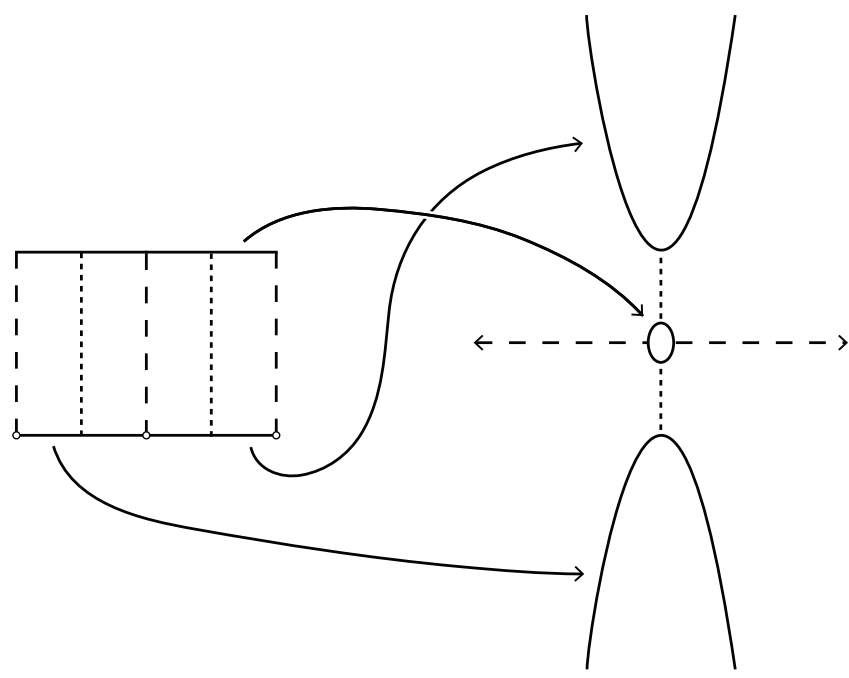

Figure 1. A doubly connected quasi-exceptional domain of type I mapped from the rectangle $G$.

This property follows from the fact that $B(z)$ and $B\left(z+\omega_{1}\right)$ have the same poles, but the residues at these poles are of opposite signs, because $B$ has only two poles in the period parallelogram. Thus

$$
B\left(z+\omega_{1}\right)=-B(z) .
$$

Property (20) and $\omega_{1}$-periodicity of $\wp$ imply (19).

As $F$ has no zeros, the primitive $f=\int F$ is locally univalent. Assuming for the moment that it is univalent, it maps $G$ onto some region in the plane, and we have

$$
\left|f^{\prime}\right|=|F|=\left|\frac{\partial v}{\partial z}\right||B| .
$$

Define $u$ by composing $v$ with $f^{-1}$, so $u(f(z))=v(z)$. Then $u$ is positive and harmonic in $f(G)$. Taking into account (18), we conclude that $u$ satisfies (1) so $f(G)$ is a quasi-exceptional domain. Note that, in accordance with the previous results in [Traizet 2014a], $f(G)$ is not an exceptional domain since the piecewiseconstant Dirichlet data is not the same constant on each boundary component.

In order to show that $f$ is in fact univalent, it is enough to show that it is oneto-one on the horizontal sides of $G$ (since $f$ is locally univalent). To this end, we make the following claims:

Claim 1: $\operatorname{Re} f$ is increasing along the segment $\left[\omega^{\prime}, \omega^{\prime}+2 \omega\right]$ and decreasing along the segment $\left[\omega^{\prime}+2 \omega, \omega^{\prime}+4 \omega\right]$. 
Claim 2: $\operatorname{Im} f<\operatorname{Im} f\left(\omega^{\prime}\right)$ on the segment $\left(\omega^{\prime}, \omega^{\prime}+2 \omega\right]$ and $\operatorname{Im} f>\operatorname{Im} f\left(\omega^{\prime}\right)$ on the segment $\left[\omega^{\prime}+2 \omega, \omega^{\prime}+4 \omega\right)$.

Claim 3: $\operatorname{Im} f$ achieves its minimum and maximum on the segment $\left[\omega^{\prime}, \omega^{\prime}+4 \omega\right]$ at $\omega^{\prime}+\omega$ and $\omega^{\prime}+3 \omega$, respectively.

Claim 4: $\operatorname{Re} f$ is increasing along the segment $[0,2 \omega]$ and $\operatorname{Re} f$ is decreasing along the segment $[2 \omega, 4 \omega]$.

Claim 5: $\operatorname{Im} f$ attains its maximum on the segment $[0,2 \omega]$ at $\omega$ and its minimum on the segment $[2 \omega, 4 \omega]$ at $3 \omega$.

Claim 6: $\operatorname{Im} f(\omega)<\operatorname{Im} f\left(\omega^{\prime}+\omega\right)<\operatorname{Im} f\left(\omega^{\prime}+3 \omega\right)<\operatorname{Im} f(3 \omega)$.

Claim 1 implies that $\operatorname{Re} f$ is monotone along each of the named segments, and since $\operatorname{Im} f$ differs between the two segments by Claim $2, f$ must be one-to-one on the top side of $G$. Claim 4 implies that $f$ is one-to-one on each of the two segments on the bottom side of $G$. Claims 3, 5, and 6 imply that the images of these three segments do not intersect each other. This shows that $f$ is one-to-one on the horizontal sides of $G$.

The claims can be established by the properties of $f^{\prime}=F=\partial_{z} v B$. First note that, since $v(z)$ is positive in $G$ and vanishes on the horizontal sides of $G$, we have $\partial_{x} v(z)=0$ on both sides, and for $x \in \mathbb{R}$ we have $\partial_{y} v\left(x+\omega_{3}\right)<0$ and $\partial_{y} v(x)>0$. In particular, $i \partial_{z} v(z)=i\left(\partial_{x} v-i \partial_{y} v\right) / 2=\partial_{y} v / 2$ is real. The function $B(z)$ is a Jacobi sn function, whose properties are well known [Akhiezer 1990, Section 47]. $B(z)$ sends the top side of $G$ to the unit circle, such that the four segments $\left[\omega^{\prime}, \omega^{\prime}+\omega\right]$, $\left[\omega^{\prime}+\omega, \omega^{\prime}+2 \omega\right],\left[\omega^{\prime}+2 \omega, \omega^{\prime}+3 \omega\right]$, and $\left[\omega^{\prime}+3 \omega, \omega^{\prime}+4 \omega\right]$ correspond to the fourth, third, second, and first quadrants of the unit circle, respectively. Multiplication by $\partial_{z} v(z)$ distorts this circle and rotates it by an angle of $\pi / 2$ (since $\partial_{z} v(z) / i$ is positive), but preserves the two-fold symmetry. This determines the sign of the real and imaginary parts of $f^{\prime}$. Since $d z=d x$ is purely real on the horizontal sides of $G$, this gives the monotonicity of $\operatorname{Re} f$ stated in Claim 1. Claims 2 and 3 follow from the sign of $\operatorname{Im} f^{\prime}$ and the fact that $\operatorname{Im} f^{\prime}$ is an odd function with respect to reflection in each of the points $\omega^{\prime}+\omega$ and $\omega^{\prime}+3 \omega$.

The four segments $[0, \omega],[\omega, 2 \omega],[2 \omega, 3 \omega]$, and $[3 \omega, 4 \omega]$ on the bottom side of $G$ are sent to the second, first, fourth, and third quadrants of the unit circle, respectively. Since $\partial_{z} v(z) / i$ is negative along the bottom side of $G$, under $f^{\prime}(z)$ this becomes the first, fourth, third, and second quadrants, respectively. This establishes Claim 4, and, combined with the reflectional symmetry, also Claim 5. Claim 6 follows from the fact that $\partial_{z} v(z) B(z)>0$ along the vertical segment $\left[\omega, \omega+\omega^{\prime}\right]$ and $\partial_{z} v(z) B(z)<0$ along $\left[3 \omega, 3 \omega+\omega^{\prime}\right]$. 


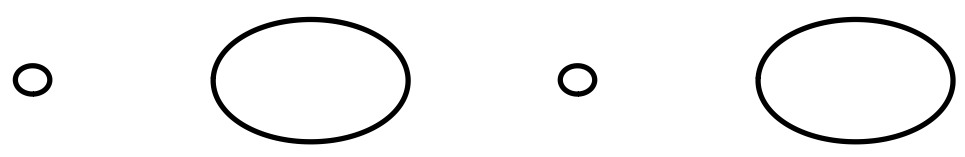

Figure 2. An example of type II with $\omega_{1}=2, \omega_{3}=2$ and $\epsilon=0.5$.
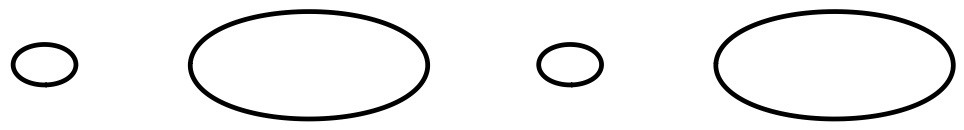

Figure 3. An example of type II with $\omega_{1}=2, \omega_{3}=1.5$, and $\epsilon=0.4$.

Remark. For the purpose of plotting Figure 1, instead of the above construction, we expressed $F$ as a ratio of Weierstrass sigma functions:

$$
f^{\prime}(z)=F(z)=\frac{\sigma\left(z-\omega+\omega^{\prime} / 2\right)^{2} \cdot \sigma\left(z-3 \omega+\omega^{\prime} / 2\right)^{2}}{\sigma(z)^{2} \cdot \sigma(z-2 \omega) \cdot \sigma\left(z-6 \omega+2 \omega^{\prime}\right)},
$$

where $\sigma$ is a Weierstrass sigma function with fundamental "periods" $4 \omega, 2 \omega^{\prime}$ (but recall that $\sigma$ is not itself periodic). As usual, the shifts are chosen based on the zeros and poles of $F$, but one of the shifts must be replaced by an equivalent lattice point in a different rectangle in order to satisfy [Akhiezer 1990, Section 14, (1)]. This explains why one of the poles is placed at $6 \omega-2 \omega^{\prime}$.

Example of type II. Only small modifications of the previous example are needed. Using the same $G, G^{\prime}, \omega_{1}, \omega_{3}$, we define $h$ as the $\omega_{1}$-periodic function, positive and harmonic in $G^{\prime}$ except two logarithmic poles at $i \epsilon$ and $\omega_{1}+i \epsilon$, where $\epsilon \in\left(0, \omega_{3} / 2\right)$. Then we can find constants $c_{1}$ and $c_{2}$ such that $v=h+c_{1} y+c_{2}$ has critical points at $\omega_{1} / 2+\omega_{3} / 2$ and $3 \omega_{1} / 2+\omega_{3} / 2$.

Then $v_{z}$ is an elliptic function with periods $\omega_{1}, 2 \omega_{3}$ with two simple poles at $i \epsilon$ and $-i \epsilon$ per period parallelogram. This elliptic function has the form

$$
\frac{-i \wp}{1+c \wp}+i c_{0}
$$

for some small real $c$. The rest of the construction is the same as in the previous example.

In a similar manner to the above, in order to plot Figures 2 and 3, we expressed $F$ as a ratio of Weierstrass sigma functions:

$$
f^{\prime}(z)=F(z)=\frac{\sigma\left(z-\omega+\omega^{\prime} / 2\right)^{2} \cdot \sigma\left(z-3 \omega+\omega^{\prime} / 2\right)^{2}}{\sigma(z-i \epsilon) \cdot \sigma(z+i \epsilon) \cdot \sigma(z-2 \omega-i \epsilon) \cdot \sigma\left(z-6 \omega+i \epsilon+2 \omega^{\prime}\right)} .
$$

Note that we have displayed the figures horizontally in order to plot two periods. 


\section{Hollow vortex equilibria}

Let $G_{j}$ be smooth Jordan domains on the plane whose closures are disjoint, and

$$
D=\mathbb{C} \backslash \bigcup_{j} G_{j}
$$

Let $F$ be the complex potential of a flow of an ideal fluid which is divergence-free and locally irrotational in $D$. If the pressure (determined by $\left|F^{\prime}\right|$ according to Bernoulli's law) is constant on $\partial D$ then $G_{j}$ can be interpreted as constant-pressure gas bubbles in the flow.

The first examples of this situation, with two bubbles, were constructed by Pocklington [1895]. Periodic exceptional domains give periodic examples with one bubble per period, with the flow on the surface on the bubbles rotating in the same direction [Baker et al. 1976] (see also [Crowdy and Green 2011]). Crowdy and Green [2011] constructed periodic examples with two bubbles per period rotating in the opposite direction. Our example of type II can be interpreted as a periodic flow with two bubbles per period rotating in the same direction.

The velocity at infinity in our examples is directed in the opposite directions on the two sides of the row of bubbles.

\section{Acknowledgments}

We are grateful to Dmitry Khavinson for many helpful discussions and to Razvan Teodorescu for a crucial observation regarding the construction of the examples of type I. We wish to thank Darren Crowdy for discussing with us the interesting connection to the hollow vortex problem. We also thank the referee who made many remarks which improved the paper.

\section{References}

[Akhiezer 1990] N. I. Akhiezer, Elements of the theory of elliptic functions, Translations of Mathematical Monographs 79, American Mathematical Society, Providence, RI, 1990. MR 91k:33016 Zbl 0694.33001

[Baker et al. 1976] G. R. Baker, P. G. Saffman, and J. S. Sheffield, "Structure of a linear array of hollow vortices of finite cross-section”, J. Fluid Mech. 74:3 (1976), 469-476. Zbl 0343.76004

[Brelot 1971] M. Brelot, On topologies and boundaries in potential theory, Lecture Notes in Mathematics 175, Springer, Berlin, 1971. MR 43 \#7654 Zbl 0222.31014

[Carleman 1926] T. Carleman, "Extension d'un théorème de Liouville", Acta Math. 48:3-4 (1926), 363-366. MR 1555232 JFM 52.0316.02

[Crowdy and Green 2011] D. G. Crowdy and C. C. Green, "Analytical solutions for von Kármán streets of hollow vortices”, Phys. Fluids 23:12 (2011), Article ID \#126602.

[Gustafsson 1987] B. Gustafsson, "Application of half-order differentials on Riemann surfaces to quadrature identities for arc-length”, J. Analyse Math. 49 (1987), 54-89. MR 89b:30032 Zbl 0652.30029 
[Hauswirth et al. 2011] L. Hauswirth, F. Hélein, and F. Pacard, "On an overdetermined elliptic problem”, Pacific J. Math. 250:2 (2011), 319-334. MR 2012g:58046 Zbl 1211.35207

[Heins 1950] M. Heins, "A lemma on positive harmonic functions", Ann. of Math. (2) 52 (1950), 568-573. MR 12,259b Zbl 0045.18803

[Khavinson et al. 2013] D. Khavinson, E. Lundberg, and R. Teodorescu, "An overdetermined problem in potential theory", Pacific J. Math. 265:1 (2013), 85-111. MR 3095114 Zbl 1279.31003

[Kjellberg 1950] B. Kjellberg, "On the growth of minimal positive harmonic functions in a plane region”, Ark. Mat. 1 (1950), 347-351. MR 12,410f Zbl 0040.05502

[Levin 1980] B. Y. Levin, Distribution of zeros of entire functions, Translations of Mathematical Monographs 5, American Mathematical Society, Providence, RI, 1980. Revised edition of 1964 original. MR 81k:30011 Zbl 0152.06703

[Longuet-Higgins 1988] M. S. Longuet-Higgins, "Limiting forms for capillary-gravity waves", J. Fluid Mech. 194 (1988), 351-375. MR 90f:76026 Zbl 0649.76005

[Pocklington 1895] H. C. Pocklington, "The configuration of a pair of equal and opposite hollow straight vortices, of finite cross-section, moving steadily through fluid", Proc. Camb. Philos. Soc. 8 (1895), 178-187. JFM 25.1468.02

[Rashkovskii 2009] A. Rashkovskii, "Classical and new loglog-theorems", Expo. Math. 27:4 (2009), 271-287. MR 2010j:31001 Zbl 1177.31001

[Sakai 1981] M. Sakai, "Null quadrature domains", J. Anal. Math. 40 (1981), 144-154. MR 84e:30069 Zbl 0483.30002

[Traizet 2014a] M. Traizet, "Classification of the solutions to an overdetermined elliptic problem in the plane", Geom. Funct. Anal. 24:2 (2014), 690-720. MR 3192039 Zbl 1295.35344

[Traizet 2014b] M. Traizet, "Hollow vortices and minimal surfaces", preprint, 2014. arXiv 1407.5308

Received June 4, 2014. Revised November 6, 2014.

ALEXANDRE EREMENKo

DEPARTMENT OF MATHEMATICS

PURDUE UNIVERSITY

150 N. UNIVERSITY STREET

WEST LAFAYETTE, IN 47907-2067

UNITED STATES

eremenko@math.purdue.edu

ERIK LUNDBERG

DEPARTMENT OF MATHEMATICAL SCIENCES

FLORIDA ATLANTIC UNIVERSITY

777 GLADES ROAD

BOCA RATON, FL 33431

UNITED STATES

elundber@fau.edu 


\title{
PACIFIC JOURNAL OF MATHEMATICS
}

\author{
msp.org/pjm
}

Founded in 1951 by E. F. Beckenbach (1906-1982) and F. Wolf (1904-1989)

\section{EDITORS}

Don Blasius (Managing Editor)

Department of Mathematics

University of California

Los Angeles, CA 90095-1555

blasius@math.ucla.edu

\author{
Paul Balmer \\ Department of Mathematics \\ University of California \\ Los Angeles, CA 90095-1555 \\ balmer@math.ucla.edu \\ Robert Finn \\ Department of Mathematics \\ Stanford University \\ Stanford, CA 94305-2125 \\ finn@math.stanford.edu \\ Sorin Popa \\ Department of Mathematics \\ University of California \\ Los Angeles, CA 90095-1555 \\ popa@math.ucla.edu
}

\author{
Vyjayanthi Chari \\ Department of Mathematics \\ University of California \\ Riverside, CA 92521-0135 \\ chari@math.ucr.edu \\ Kefeng Liu \\ Department of Mathematics \\ University of California \\ Los Angeles, CA 90095-1555 \\ liu@math.ucla.edu \\ Jie Qing \\ Department of Mathematics \\ University of California \\ Santa Cruz, CA 95064 \\ qing@ cats.ucsc.edu
}

\section{PRODUCTION}

Silvio Levy, Scientific Editor, production@msp.org

\section{SUPPORTING INSTITUTIONS}

ACADEMIA SINICA, TAIPEI

CALIFORNIA INST. OF TECHNOLOGY

INST. DE MATEMÁTICA PURA E APLICADA

KEIO UNIVERSITY

MATH. SCIENCES RESEARCH INSTITUTE

NEW MEXICO STATE UNIV.

OREGON STATE UNIV.

\author{
STANFORD UNIVERSITY \\ UNIV. OF BRITISH COLUMBIA \\ UNIV. OF CALIFORNIA, BERKELEY \\ UNIV. OF CALIFORNIA, DAVIS \\ UNIV. OF CALIFORNIA, LOS ANGELES \\ UNIV. OF CALIFORNIA, RIVERSIDE \\ UNIV. OF CALIFORNIA, SAN DIEGO \\ UNIV. OF CALIF., SANTA BARBARA
}

\author{
Daryl Cooper \\ Department of Mathematics \\ University of California \\ Santa Barbara, CA 93106-3080 \\ cooper@math.ucsb.edu \\ Jiang-Hua Lu \\ Department of Mathematics \\ The University of Hong Kong \\ Pokfulam Rd., Hong Kong \\ jhlu@maths.hku.hk \\ Paul Yang \\ Department of Mathematics \\ Princeton University \\ Princeton NJ 08544-1000 \\ yang@math.princeton.edu
}

These supporting institutions contribute to the cost of publication of this Journal, but they are not owners or publishers and have no responsibility for its contents or policies.

See inside back cover or msp.org/pjm for submission instructions.

The subscription price for 2015 is US \$420/year for the electronic version, and \$570/year for print and electronic.

Subscriptions, requests for back issues and changes of subscribers address should be sent to Pacific Journal of Mathematics, P.O. Box 4163, Berkeley, CA 94704-0163, U.S.A. The Pacific Journal of Mathematics is indexed by Mathematical Reviews, Zentralblatt MATH, PASCAL CNRS Index, Referativnyi Zhurnal, Current Mathematical Publications and Web of Knowledge (Science Citation Index).

The Pacific Journal of Mathematics (ISSN 0030-8730) at the University of California, c/o Department of Mathematics, 798 Evans Hall \#3840, Berkeley, CA 94720-3840, is published twelve times a year. Periodical rate postage paid at Berkeley, CA 94704, and additional mailing offices. POSTMASTER: send address changes to Pacific Journal of Mathematics, P.O. Box 4163, Berkeley, CA 94704-0163.

PJM peer review and production are managed by EditFLOW ${ }^{\circledR}$ from Mathematical Sciences Publishers.

\section{PUBLISHED BY}

\section{mathematical sciences publishers \\ nonprofit scientific publishing}

http://msp.org/

(C) 2015 Mathematical Sciences Publishers 


\section{PACIFIC JOURNAL OF MATHEMATICS}

Volume $276 \quad$ No. $1 \quad$ July 2015

On the degree of certain local $L$-functions

U. K. ANANDAVARdhanan and AmiYa Kumar MondaL

Torus actions and tensor products of intersection cohomology

ASILATA BAPAT

Cyclicity in Dirichlet-type spaces and extremal polynomials II: functions on the bidisk

Catherine Bénéteau, Alberto A. Condori, Constanze Liaw,

DANIEl SECo and Alan A. SOlA

Compactness results for sequences of approximate biharmonic maps

CHRISTINE BREINER and TOBIAS LAMM

Criteria for vanishing of Tor over complete intersections

Olgur Celikbas, Srikanth B. Iyengar, Greg PiePMeyer and

ROGER WIEGAND

Convex solutions to the power-of-mean curvature flow

\section{SHIBING CHEN}

Constructions of periodic minimal surfaces and minimal annuli in $\mathrm{Sol}_{3}$

CHRISTOPHE DESMONTS

Quasi-exceptional domains

ALEXANDRE EREMENKO and ERIK LUNDBERG

Endoscopic transfer for unitary groups and holomorphy of Asai $L$-functions

NEVEN GRBAC and FREYDOON SHAHIDI

Quasiconformal harmonic mappings between Dini-smooth Jordan domains

DAVID KALAJ

Semisimple super Tannakian categories with a small tensor generator

THOMAS KRÄMER and RAINER WEISSAUER

On maximal Lindenstrauss spaces

PETR PETRÁČEK and JiŘí SPURNÝ 